CLINICAL STUDY

\title{
The risk of fractures in postmenopausal women with primary hyperparathyroidism
}

\author{
Simona De Geronimo*, Elisabetta Romagnoli*, Daniele Diacinti, Emilio D’Erasmo and Salvatore Minisola \\ Department of Clinical Sciences, University of Rome 'La Sapienza', Viale del Policlinico 155, 00161 Rome, Italy \\ (Correspondence should be addressed to E Romagnoli; Email: elisabetta.romagnoli@libero.it)
}

*(S De Geronimo and E Romagnoli contributed equally to this work)

\begin{abstract}
Objective: To evaluate the prevalence of vertebral ( $\mathrm{vFr})$ and non-vertebral (nvFr) fractures in postmenopausal women with primary hyperparathyroidism (PHPT).

Materials and Methods: We studied 98 patients with PHPT, divided into 'mild' (M, $n=25)$ and "nonmild' (NM, $n=73$ ) sub-groups, according to recently published guidelines (2002), and 89 healthy women $(C)$ matched for age, years since menopause and body mass index. vFr was evaluated according to a visual semiquantitative method; bone mineral density (BMD) at the lumbar spine (LS), and femoral sites (femoral neck, FN and total femur, FT) was measured by dual energy X-ray absorptiometry. Volumetric BMD of the third lumbar vertebra (vBMDL3) was also calculated.

Results: The prevalence of vFr was significantly higher $(P<0.001)$ in both M and NM PHPT patients compared with $C$; this prevalence did not differ between $\mathrm{M}$ and NM patients. BMD was significantly lower $(P<0.05)$ in NM patients compared with both $\mathrm{C}$ and $\mathrm{M}$ patients. BMD at LS in $\mathrm{M}$ patients was also significantly higher with respect to $C$. Similar results were also obtained for vBMDL3; in M patients, vBMDL3 was also significantly higher compared to $\mathrm{C}$. When $\mathrm{M}$ and NM patients were subdivided according to the presence or lack of vFr, no difference was found between fractured and unfractured patients for either BMD or vBMDL3 values.

Conclusions: The risk of vFr is higher in postmenopausal patients with mild PHPT even if BMD appears well preserved. This finding suggests that other factors, such as bone quality, seem to be relevant in determining fracture risk, especially when gonadal function is lacking.
\end{abstract}

European Journal of Endocrinology 155 415-420

\section{Introduction}

Fracture risk in patients with asymptomatic primary hyperparathyroidism (PHPT) is still a controversial issue, which is difficult to work out on the basis of available data $(1,2)$. Based upon the densitometric and histomorphometric findings, it may be expected that the cortical skeleton would be at greater risk of fracture with respect to the cancellous skeleton $(3,4)$. In fact, it has been shown that bone mineral density (BMD) is lower at the cortical site, whereas bone mass is relatively well preserved in trabecular bone (5). Similarly, bone biopsy shows cortical thinning but maintenance of cancellous bone volume (6). These findings suggest that the effect of endogenous parathyroid hormone (PTH) excess is different in trabecular and cortical bones (7). However, a discrepancy between BMD measurement and histology on one hand, and the distribution of fractures on the other had been demonstrated by several retrospective and case-control studies. In fact, some authors have found no increase in the risk of appendicular or vertebral fractures $(8,9)$, whereas others have (10-13). Methodological problems could be partially responsible for these conflicting results, since many studies are limited by their design, inadequate control groups, ascertainment biases and, most importantly, imprecise definitions of fracture. This is particularly true regarding the definition of vertebral fracture. In fact, several epidemiological studies have demonstrated that the majority of these fractures are asymptomatic (14); many cases may be treated as 'simple' back pain by general practitioners and X-rays may never be taken. However, morphometric vertebral deformities also increase the risk of both vertebral and nonvertebral fractures and are associated with significant morbidity and mortality (15). The aim of this study was to evaluate the prevalence of vertebral and appendicular fractures in a group of 98 postmenopausal women with PHPT. Female patients should be at higher risk of vertebral fractures because estrogen deficiency would be expected to be associated with the preferential reduction of cancellous bone. If the risk of fractures, particularly subclinical vertebral fractures, is 
higher in patients with PHPT, then the evaluation of skeletal involvement, as is presently proposed, needs to be revised.

\section{Materials and methods}

Ninety-eight consecutive postmenopausal patients with PHPT (age (mean \pm s.D.), $61.44 \pm 8.0$ years; years since menopause (YSM), 13.18 \pm 9.62 ), from our Mineral Metabolism Center, were enrolled in the study. They were grouped as 'mild' (M) or 'non-mild' (NM) according to the criteria established by the Consensus Development Conference on the Management of Asymptomatic Primary Hyperparathyroidism, recently reviewed by Bilezikian et al. (16) as follows: (i) serum calcium greater than $1 \mathrm{mg} / \mathrm{dl}$ above the upper limits of normal; (ii) 24-h total urine calcium excretion of more than $400 \mathrm{mg}$; (iii) creatinine clearance reduced by more than 30\% compared to age-matched persons; (iv) bone density at the lumbar spine (LS), hip or distal radius that is more than 2.5 s.D. below peak bone mass ( $T$ score $<-2.5$ ) (however, for technical reasons we were not able to measure the forearm BMD at the start of the study); and (v) patients under 50 years of age. Based on these widely accepted criteria, only 25 of the 98 patients were considered as suffering from mild disease. In particular, in this group of patients, hypercalcemia was occasionally detected in the course of the standard biochemical evaluation performed in our center on all subjects undergoing BMD measurement. None of the $\mathrm{M}$ patients reported either height loss or clinical vertebral fractures at history. In the remaining 73 patients, a severe disease was present; in particular, 13 of them had a history of clinical nonvertebral fractures for minimal/moderate trauma (by convention, falls from standing height or less were considered moderate trauma); 7 and 21 patients were referred to our center by their general practitioners for a comprehensive clinical evaluation of nephrolithiasis and osteoporosis respectively; in 15 patients renal stones were shown by ultrasonography; three were referred for etiologic definition of hypercalcemia; two had a life-threatening episode of pancreatitis. The remaining 12 patients complained of bone pain and/or neuromuscular symptoms. Diagnosis of PHPT was made according to the conventional clinical and laboratory data, including a history of at least 1 year of prolonged hypercalcemia without evidence of a nonparathyroid etiology and unsuppressed serum levels of immunoreactive PTH. Familial hypocalciuric hypercalcemia was excluded because in all cases the ratio of calcium to creatinine clearance was $>0.01$. Clinical diagnosis was confirmed in 61 patients during surgery and by histological examination (removal of a parathyroid adenoma). During the period of the study, no patients took medications (including estrogen) known to influence mineral metabolism.
Eighty-nine healthy postmenopausal women, matched for age, YSM and body mass index (BMI) were concomitantly studied as a control group (C). They were randomly selected from ambulatory postmenopausal women sent by their general practitioners to our hospital as a part of a menopause-screening program. In each woman, medical history, physical examination, and laboratory tests excluded disorders of bone and mineral metabolism. All patients and normal subjects had standardized lateral radiographs in antero-posterior and left lateral projections of the thoracic and LS, centered at T8 and L3 respectively, at a film-focus distance of $115 \mathrm{~cm}$. The radiographs were examined first for quality and then for fractures by an experienced skeletal radiologist. Vertebral deformity was defined, according to the visual semiquantitative method, when anterior, middle, or posterior height loss was more than $20 \%$ with respect to the adjacent vertebra (17). This criterion for defining vertebral fracture had a relatively high true-positive rate and low false-positive rate based on the classifications from previous reports (18). None of the patients or normal subjects showed evidence of fracture of the third lumbar vertebra. BMD at the LS in posterior-anterior projection (L1-L4) (LS-BMD) and femoral sites (femoral neck, FN-BMD and total hip, FT-BMD) was measured in each patient and healthy subject by dual energy X-ray absorptiometry (DEXA) technique using a QDR 4500A (Hologic, Inc., Bedford, MA, USA). In order to recognize the influence of bone size on bone fragility, we also calculated volumetric BMD of the third lumbar vertebra (vBMD-L3) according to the method described by Duan and coworkers (19). Vertebral body volume $(V)$ was estimated as $V=A^{3 / 2}$, where $A$ is the projected area of the third lumbar vertebra obtained by posterior-anterior scanning; $\mathrm{vBMD}=\mathrm{BMC} / V$. The metabolic study included a 24-h urine collection, followed by a fasting short urine collection (from 0800 to $1100 \mathrm{~h}$ ) along with a blood sample, in order to measure the main parameters of calcium metabolism, as well as PTH and 25-hydroxyvitamin D (250HD) serum levels, according to the method described previously (20). All patients and healthy subjects were called together for metabolic evaluation between March and May 2005, in order to minimize the seasonal variation of vitamin D status. The study was approved by the local Ethics Committee. All subjects gave informed consent.

\section{Statistical analysis}

Descriptive statistics are expressed as the mean \pm s.D. for each index. After a test for normality, comparisons between groups were performed by $t$-test or one-way ANOVA, as appropriate. Differences between frequencies were assessed by $\chi^{2}$-analysis. Statistical significance was set at $P<0.05$. 


\section{Results}

Main demographic and biochemical variables observed in all the groups examined are reported in Table 1. As can be seen, no difference was found between $\mathrm{M}$ and NM PHPT patients and C in age, YSM, height, and BMI. As expected, in NM patients, serum total and ionized calcium, PTH levels and fasting urinary calcium/ creatinine excretion were significantly higher compared with $\mathrm{M}$ patients. Renal function was also reduced in NM patients. However, serum levels of $250 \mathrm{HD}$ did not differ among the three groups. Values regarding BMD, vBMD$\mathrm{L} 3$, and related variables (bone mineral content, BMC and volume) are reported in Table 2. As shown, in $\mathrm{M}$ patients, BMD values were higher compared with $C$; this difference was significant for LS-BMD $(P<0.05)$. Moreover, $\mathrm{M}$ patients show significantly higher values of vBMD-L3 with respect to $C$ because of a significant increase in BMC $(P<0.05)$. In NM patients, BMD at any site and $\mathrm{VBMD}$ were significantly lower with respect to both $\mathrm{M}$ patients and C. Furthermore, when classifying patients by quartiles of PTH serum levels, vBMD values significantly decreased from the lowest to the highest quartile of PTH ( $P=0.033$ by ANOVA). In Table 3, the distribution of vertebral $(\mathrm{vFr})$ and non-vertebral $(\mathrm{nvFr})$ fractures in the two subgroups of patients is reported, compared with that found in healthy women. M patients had a significantly higher percentage of $\mathrm{vFr}$ $(44 \%)$ than $\mathrm{C}(P<0.001)$. The percentage of $\mathrm{vFr}$ was also significantly higher in NM patients compared with C $(47$ vs $9 \%, P<0.001)$. However, the percentage of vertebral fractures did not differ between $\mathrm{M}$ and NM patients. As far as nvFr are concerned, no difference was found between NM patients and C. When M and NM patients were subdivided according to the presence or lack of vertebral fractures, no difference was found between fractured and unfractured patients in BMD, vBMD-L3, and related variables (Table 4). However, as far as M patients are concerned, those without vertebral fractures show significantly higher values of LS-BMD, $\mathrm{BMC}$, and $\mathrm{vBMD}$ compared with $\mathrm{C}$; on the contrary, these variables were not different from $\mathrm{C}$ in $\mathrm{M}$ patients with vFr. In patients with more severe disease, independent of the presence or absence of vertebral fractures, BMD at both LS and femoral sites was significantly lower compared with C. However, only in fractured NM patients were BMC and $\mathrm{VBMD}$ values significantly lower than in $\mathrm{C}$.

\section{Discussion}

Few studies have focused on the issue of fracture risk in patients with PHPT. Patients with mild disease should have a reduction in the risk of vertebral fractures due to the anabolic action of the PTH in the cancellous bone. On the contrary, appendicular fractures should be more common, since PTH is catabolic for the cortical skeleton (21). However, these findings are not supported by the two most important epidemiological studies on fracture risk: the study of Koshla et al. (11), which enrolled patients with mild PHPT, and the study of Vestergaard and coworkers (10), which was carried out on patients before and after parathyroidectomy, both demonstrated an increase in fracture risk at all the examined sites. Several explanations are plausible for this unexpected increase in vertebral fractures: (i) surveillance bias; (ii) thinning of the cortical rim of the vertebral bodies; (iii) generally higher bone turnover; and (iv) inclusion of patients with lower LS BMD, thus at higher risk. Our study focused on postmenopausal women with PHPT in which, fracture risk should be higher and the disease

Table 1 Main demographic and biochemical variables of healthy postmenopausal women and patients grouped according to the severity of the disease.

\begin{tabular}{|c|c|c|c|c|}
\hline & Healthy women $(C)$ & Mild PHPT patients (M) & $\begin{array}{l}\text { Non-mild PHPT } \\
\text { patients (NM) }\end{array}$ & $\boldsymbol{P}^{*}$ \\
\hline$n$ & 89 & 25 & 73 & \\
\hline Age (years) & $60.65 \pm 6.92$ & $60.84 \pm 6.82$ & $61.65 \pm 8.40$ & n.s. \\
\hline YSM (years) & $11.15 \pm 8.99$ & $13.44 \pm 10.44$ & $13.09 \pm 9.40$ & n.s. \\
\hline Height $(\mathrm{cm})$ & $159.1 \pm 5.9$ & $161.6 \pm 6.1$ & $159.9 \pm 6.3$ & n.s. \\
\hline BMI & $24.8+3.1$ & $26.2+3.5$ & $25.1+3.9$ & n.s. \\
\hline $\mathrm{sCa}(\mathrm{mg} / \mathrm{dl})$ & $9.53 \pm 0.51$ & $10.66 \pm 0.39 t$ & $11.14 \pm 0.96 † \ddagger$ & $<0.001$ \\
\hline $\mathrm{iCa}(\mathrm{mmol} / \mathrm{l})$ & $1.24 \pm 0.02$ & $1.44 \pm 0.06 \dagger$ & $1.50 \pm 0.145 \dagger \ddagger$ & $<0.001$ \\
\hline $\mathrm{P}(\mathrm{mg} / \mathrm{dl})$ & $3.64 \pm 0.49$ & $2.92 \pm 0.38 \dagger$ & $2.81 \pm 0.56 \dagger$ & $<0.001$ \\
\hline $\operatorname{ALP}(U / I)$ & $74.04 \pm 19.49$ & $106.96 \pm 28.49 \S$ & $171.89 \pm 279.19 \S$ & $<0.001$ \\
\hline $\mathrm{sCr}(\mathrm{mg} / \mathrm{dl})$ & $0.84+0.13$ & $0.81+0.13$ & $0.95+0.45$ & n.s. \\
\hline $\mathrm{CICr}(\mathrm{ml} / \mathrm{min})$ & $90.3 \pm 18.7$ & $82.8 \pm 20.2$ & $69.7 \pm 22.4 \S \ddagger$ & $<0.001$ \\
\hline $25 \mathrm{OHD}(\mathrm{ng} / \mathrm{ml})$ & $19.1 \pm 9.5$ & $18.4 \pm 5.8$ & $18.0 \pm 8.7$ & n.s. \\
\hline PTH $(\mathrm{pg} / \mathrm{ml})$ & $28.8 \pm 9.3$ & $82.9 \pm 36.7 \S$ & $168.5 \pm 211.1 \S \ddagger$ & $<0.001$ \\
\hline u Fasting $\mathrm{Ca} / \mathrm{Cr}(\mathrm{mg} / \mathrm{mg})$ & $150.3 \pm 80.5$ & $183.0 \pm 97.4$ & $263.3 \pm 149.6 \S \ddagger$ & $<0.001$ \\
\hline u Ca (mg/24h) & $158.7 \pm 83.0$ & $228.1 \pm 90.9 \S$ & $286.7 \pm 135.5 \S$ & $<0.001$ \\
\hline
\end{tabular}

YSM, years since menopause; BMI, body mass index; s, serum; i, ionized; Ca, calcium; P, phosphorus, ALP, alkaline phosphatase; $\mathrm{Cr}$, creatinine; $\mathrm{Cl} / \mathrm{Cr}$, clearance creatinine; 25OHD, 25-hydroxy-vitamin D; PTH, parathyroid hormone; u, urinary. *Comparisons were performed by one-way ANOVA. §Significantly different from $\mathrm{C}, P<0.05$; †significantly different from $\mathrm{C}, P<0.001$; ‡significantly different from $\mathrm{M}$ patients, $P<0.05$. 
Table 2 Mean \pm S.D. values of bone mineral density and of volumetric bone mineral density, bone mineral content and volume of the third lumbar vertebra in healthy postmenopausal women and patients grouped according to the severity of the disease.

\begin{tabular}{|c|c|c|c|c|}
\hline & Healthy women (C) & Mild PHPT patients (M) & $\begin{array}{l}\text { Non-mild PHPT patients } \\
\text { (NM) }\end{array}$ & $P^{*}$ \\
\hline$n$ & 89 & 25 & 73 & \\
\hline LS-BMD $\left(\mathrm{mg} / \mathrm{cm}^{2}\right)$ & $839.0 \pm 109.8$ & $916.1 \pm 100.4 \dagger$ & $765.4 \pm 156.6 † \ddagger$ & $<0.001$ \\
\hline FN-BMD $\left(\mathrm{mg} / \mathrm{cm}^{2}\right)$ & $690.6 \pm 109.1$ & $709.0 \pm 92.7$ & $601.3 \pm 102.5 \dagger \ddagger$ & $<0.001$ \\
\hline FT-BMD $\left(\mathrm{mg} / \mathrm{cm}^{2}\right)$ & $802.2 \pm 116.0$ & $823.4 \pm 116.2$ & $699.7 \pm 126.0 \dagger \ddagger$ & $<0.001$ \\
\hline Volume $\left(\mathrm{cm}^{3}\right)$ & $51.22 \pm 7.91$ & $51.48 \pm 5.99$ & $51.56 \pm 9.14$ & n.s. \\
\hline $\mathrm{BMC}(\mathrm{g})$ & $11.89 \pm 2.25$ & $13.25 \pm 2.11 \dagger$ & $10.93 \pm 3.04 † \ddagger$ & $<0.001$ \\
\hline vBMD-L3 $\left(\mathrm{g} / \mathrm{cm}^{3}\right)$ & $0.235 \pm 0.031$ & $0.257 \pm 0.025 \dagger$ & $0.213 \pm 0.040 \dagger \ddagger$ & $<0.001$ \\
\hline
\end{tabular}

BMD, bone mineral density; LS, lumbar spine; FN, femoral neck; FT, total femur; v, volumetric; BMC, bone mineral content; ${ }^{\star}$ Comparisons were performed by one-way ANOVA. †Significantly different from $\mathrm{C}, P<0.05$; ‡significantly different from $\mathrm{M}$ patients, $P<0.05$.

was more frequent. We also analyzed patients grouped according to the severity of the disease, thus limiting methodological problems arising from the analysis of non-homogenous series. This also allowed us to hypothesize that an increase in fragility fracture in patients with mild PHPT should be considered as a new indication for surgery. In fact, according to 2002 guidelines, skeletal involvement in M PHPT patients is only evaluated by BMD measurement. As expected, in patients with more severe disease, BMD values were significantly lower at any site compared with both $\mathrm{M}$ patients and C. However, confirming our previous results (22), in M patients BMD values at the LS were significantly higher than $\mathrm{C}$, supporting the hypothesis of an anabolic action of PTH on cancellous bone in postmenopausal women with mild disease. To define this action better, we also calculated volumetric BMD of the third lumbar vertebra according to an accepted and widely used method (19). In fact, neither BMC nor areal $\mathrm{BMD}$, as assessed by DEXA, fully account for bone size. Bone size is an independent determinant of bone strength, which may be influenced by excessive concentrations of PTH by virtue of its role in eroding subcortical bone and stimulating periosteal bone formation $(23,24)$. Our M patients not only have higher BMD values, but also show significantly higher vBMD-L3 compared with $C$. The increase in vBMD seems to be dependent on the increase in BMC, since the volume of the vertebrae did not differ among the three groups. Interestingly, by classifying patients according to quartiles of PTH levels, we found that vBMD significantly decreased from the lowest to the highest quartile of PTH; this finding again supports the hypothesis of anabolic action of PTH on cancellous bone in mild disease. Our results are original, because very few studies have addressed the issue of volumetric BMD in patients with PHPT. In the study of Chen and coworkers (25), trabecular BMD measured by peripheral quantitative computed tomography was significantly lower in patients with PHPT compared with controls. However, in this study, no information about the severity of the disease was made and no analysis on weight-bearing bone, such as the spine, was performed.
If the relationship between bone density and fracture risk, clearly shown for postmenopausal women, is applicable in the same manner to PHPT, a reduction in vertebral fractures is expected. However, our $M$ patients show a percentage of vertebral fractures significantly higher (44\%) than C (9\%) but not different compared with that observed in patients with more severe disease $(47 \%)$. Therefore, BMD does not seem to be the only factor determining fracture risk in PHPT. In fact, bone strength is affected by BMD as well as bone quality, which includes bone structure, the accumulation of microdamage and bone turnover rate. Subdividing $\mathrm{M}$ and $\mathrm{NM}$ patients according to the presence or lack of vertebral fractures, no difference was found between fractured and unfractured patients in BMD or in variables related to volumetric BMD. Moreover, when comparing these subgroups of patients with healthy women, we observed that in fractured $\mathrm{M}$ patients, BMD values and parameters related to volumetric BMD were not different from $C$. On the contrary, in NM patients, BMD at any site was lower than in $\mathrm{C}$, independently from the presence or lack of vertebral fractures. Our data clash with those recently published by Kaji and coworkers (26), whose data demonstrate that only LS BMD was lower in postmenopausal women with PHPT and vertebral fractures. However, in this study, patients were not separated according to the severity of disease and this could have influenced the results obtained. Our data seem to suggest that in patients with PHPT, BMD measurement

Table 3 Distribution of vertebral and non-vertebral fractures in healthy postmenopausal women and patients grouped according to the severity of the disease.

\begin{tabular}{lccc}
\hline & $\begin{array}{c}\text { Healthy } \\
\text { women (C) }\end{array}$ & $\begin{array}{c}\text { Mild PHPT } \\
\text { patients (M) }\end{array}$ & $\begin{array}{c}\text { Non-mild } \\
\text { PHPT patients } \\
(\mathrm{NM})\end{array}$ \\
\hline$n$ & $\begin{array}{c}89 \\
\mathrm{vFr}\end{array}$ & 25 & 73 \\
Non-vFr & $n=8(9 \%)$ & $n=11^{\star}(44 \%)$ & $n=35^{*}(47 \%)$ \\
\hline
\end{tabular}

$\mathrm{vFr}$, vertebral fractures; $n$, number of controls and patients with fractures; in brackets percentage of controls and patients with fractures. ${ }^{\star} P<0.001$ vs controls by $\chi^{2}$-analysis. 
Table 4 Mean \pm S.D. values of bone mineral density and of volumetric bone mineral density, bone mineral content and volume of the third lumbar vertebra in healthy postmenopausal women and patients grouped according to the severity of the disease and to the presence or lack of vertebral fractures.

\begin{tabular}{|c|c|c|c|c|c|c|c|}
\hline & \multirow{2}{*}{$\begin{array}{c}\text { Healthy } \\
\text { women }(C)\end{array}$} & \multicolumn{3}{|c|}{ Mild PHPT patients (M) } & \multicolumn{3}{|c|}{ Non-mild PHPT patients (NM) } \\
\hline & & Fractured & Unfractured & $P^{*}$ & Fractured & Unfractured & $P^{*}$ \\
\hline$n$ & 89 & 11 & 14 & & 35 & 38 & \\
\hline LS-BMD (mg/cm²) & $839.0 \pm 109.8$ & $887.5 \pm 77.4$ & $938.5 \pm 113.0 \dagger$ & $<0.01$ & $761.8 \pm 144.3 \dagger$ & $768.89 \pm 168.9 \dagger$ & $<0.001$ \\
\hline FN-BMD $\left(\mathrm{mg} / \mathrm{cm}^{2}\right)$ & $690.6 \pm 109.1$ & $716.0 \pm 113.3$ & $703.5 \pm 76.8$ & n.s. & $579.6 \pm 88.1 \dagger$ & $621.3 \pm 111.6+$ & $<0.001$ \\
\hline FT-BMD (mg/cm²) & $802.2 \pm 116.0$ & $835.5 \pm 137.0$ & $813.8 \pm 101.4$ & n.s. & $681.3 \pm 111.3 \dagger$ & $716.6 \pm 137.5 \dagger$ & $<0.001$ \\
\hline BMC (g) & $11.89 \pm 2.25$ & $12.49 \pm 1.49$ & $13.83 \pm 2.38 \dagger$ & $<0.05$ & $10.46 \pm 3.37 \dagger$ & $11.35 \pm 2.71$ & $<0.05$ \\
\hline Volume $\left(\mathrm{cm}^{3}\right)$ & $51.22 \pm 7.91$ & $50.39 \pm 6.02$ & $52.31 \pm 6.08$ & n.s. & $51.47 \pm 10.50$ & $51.63 \pm 7.94$ & n.s. \\
\hline vBMD-L3 (g/cm³) & $0.235 \pm 0.031$ & $0.248 \pm 0.016$ & $0.264 \pm 0.029 \dagger$ & $<0.01$ & $0.203 \pm 0.049 \dagger$ & $0.221 \pm 0.043$ & $<0.001$ \\
\hline
\end{tabular}

BMD, bone mineral density; LS, lumbar spine; FN, femoral neck; FT, total femur; v, volumetric; BMC, bone mineral content; *Comparisons were performed by one-way ANOVA. †Significantly different from $\mathrm{C}, P<0.05$.

is not specific to fracture sites and a different relationship exists between fracture risk and BMD. Furthermore, data on volumetric BMD found in $\mathrm{M}$ patients should be consulted. In fact, it has been demonstrated that women with spine fractures have smaller vertebrae with less bone in the smaller bone (19). On the contrary, in our sample, fractured M patients show both BMD and vBMD values not different from $C$; furthermore, these parameters were found to be increased in unfractured $M$ patients. This finding further supports the anabolic action of the PTH in mild disease and the fact that other factors could be relevant in determining fracture risk in PHPT. For example, it is now known that PTH may increase the cross-sectional area of bone and therefore strengthen it, despite a reduction in BMD (27). We found that in $\mathrm{M}$ patients, no appendicular fractures were registered, while in NM patients, the percentage of these fractures was not higher compared with $\mathrm{C}$, even if BMD was lower. Data on the risk of non-vertebral fractures in patients with PHPT are uncertain, perhaps because fractures are not always classified according to the circumstances of the injury and, therefore, also traumatic fractures may be included in some case studies. Moreover, metabolic parameters related to the disease could modify skeletal (28) as well as non-skeletal risk factors for fractures, such as the tendency to fall. Regarding this, it is now known that vitamin D influences not only bone metabolism, but also muscle strength and function (29). We found that serum levels of $250 H D$ were similar among the three groups studied. Moreover, confirming our previous results (20), no correlations were found between $250 \mathrm{HD}$ serum levels and BMD in both M and NM patients (data not shown). Therefore, we can hypothesize that, at least in our population, the susceptibility to falls is not different between patients and controls. There are some limitations in the present study. First, compared with other case studies, the proportion of $\mathrm{M}$ patients is smaller (25 of 98 patients); since our patients were recruited from a referring center for osteoporosis and do not represent the general population, some selection bias might be included in the cases. Secondly, for technical reasons we were not able to measure the forearm BMD at the start of the study. Therefore, we may have underestimated the proportion of patients with osteoporosis and their need for parathyroidectomy according to the current criteria. However, according to Kaji's results (26), radial BMD measurement is not predictive of vertebral fracture risk in PHPT patients, so the lack of forearm BMD data should not jeopardize the main results of the study. In conclusion, the risk of vertebral fractures is higher in postmenopausal women with PHPT, independently of the severity of the disease, even if BMD appears well preserved in M patients. These findings strongly indicate that X-ray examination of the thoracic and LS is mandatory, especially in M patients. Our results also suggest that other factors, such as bone quality, seem to be relevant in determining fracture risk. Finally, the current guidelines for surgery in asymptomatic patients based only on the T-score may be considered insufficient; the evaluation of vertebral fractures could be proposed among the criteria for parathyroidectomy in these patients.

\section{Acknowledgements}

We thank Ms Lee Tanya O’Hara for editorial assistance.

\section{References}

1 Khan A \& Bilezikian J. Primary hyperparathyroidism: pathophysiology and impact on bone. Canadian Medical Association Journal $2000163184-187$.

2 Cormier C, Souberbielle JC \& Kahan A. Primary hyperparathyroidism and osteoporosis in 2004. Joint Bone Spine 200471 183-189.

3 Bilezikian JP, Rubin M \& Silverberg SJ. Primary hyperparathyroidism: diagnosis, evaluation, and treatment. Current Opinion in Endocrinology and Diabetes 200411 345-352.

4 Bilezikian JP, Brandi ML, Rubin M \& Silverberg SJ. Primary hyperparathyroidism: new concepts in clinical, densitometric and biochemical features. Journal of Internal Medicine 2005257 6-17.

5 Silverberg SJ, Shane E, de la Cruz L, Dempster DW, Feldman F, Seldin D, Jacobs TP, Siris ES, Cafferty M, Parisien MV, Lindsay R, 
Clemens TL \& Bilezikian JP. Skeletal disease in primary hyperparathyroidism. Journal of Bone and Mineral Research 1989 4 283-291.

6 Dempster DW, Parisien M, Silverberg SJ, Liang X-G, Schnitzer M, Shen V, Shane E, Kimmel DB, Recker R, Lindsay R \& Bilezikian JP. On the mechanism of cancellous bone preservation in postmenopausal women with mild primary hyperparathyroidism. Journal of Clinical Endocrinology and Metabolism $1999 \mathbf{8 4} 1562-1566$.

7 Rubin MR, Cosman F, Lindsay R \& Bilezikian JP. The anabolic effects of parathyroid hormone. Osteoporosis International 200213 267-277.

8 Wilson RJ, Sudhaker R, Ellis B, Kleerekoper M \& Parfitt MA. Mild asymptomatic primary hyperparathyroidism is not a risk factor for vertebral fractures. Annals of Internal Medicine 1988109 959-962.

9 Larsson K, Ljunghall S, Krusemo UB, Naessen T, Lindh E \& Persson I. The risk of hip fractures in patients with primary hyperparathyroidism: a population-based cohort study with a follow-up of 19 years. Journal of Internal Medicine 1993234 585-593.

10 Vestergaard P, Mollerup CL, Frøkjaer VG, Christiansen P, BlichertToft M \& Mosekilde L. Cohort study of risk of fracture before and after surgery for primary hyperparathyroidism. British Medical Journal 2000321 598-602.

11 Khosla S, Melton LJ, III, Wermers RA, Crowson CS, O'Fallon WM \& Riggs BL. Primary hyperparathyroidism and the risk of fracture: a population-based study. Journal of Bone and Mineral Research 1999 14 1700-1707.

12 Vestergaard P \& Mosekilde L. Parathyroid surgery is associated with a decreased risk of hip and upper arm fractures in primary hyperparathyroidism: a controlled cohort study. Journal of Internal Medicine 2004255 108-114.

13 Kenny AM, MacGillivray DC, Pilbeam CC, Crombie HD \& Raisz LG. Fracture incidence in postmenopausal women with primary hyperparathyroidism. Surgery 1995118 109-114.

14 Black DM, Cummings SR, Karpf DB, Cauley JA, Thompson DE, Nevitt MC, Bauer DC, Genant HK, Haskell WL, Marcus R, Ott SM, Torner JC, Quandt SA, Reiss TF \& Ensrud KE. Randomised trial of the effect of alendronate on risk of fracture in women with existing vertebral fractures. Lancet 1996348 1535-1541.

15 Hasserius R, Karlsson MK, Nilsson BE, Redlund-Johnell I \& Johnell O, European Vertebral Osteoporosis Study. Prevalent vertebral deformities predict increased mortality and increased fracture rate in both men and women: a 10-year population-based study of 598 individuals from the Swedish cohort in the European Vertebral Osteoporosis Study. Osteoporosis International 200314 61-68.

16 Bilezikian JP \& Potts JT, Jr. Asymptomatic primary hyperparathyroidism: new issues and new questions-bridging the past with the future. Journal of Bone and Mineral Research 200217 N57-N67.

17 Genant HK, Wu CY, van Kuijk C \& Nevitt MC. Vertebral fracture assessment using a semiquantitative technique. Journal of Bone and Mineral Research 19938 1137-1148.
18 Genant HK \& Jergas M. Assessment of prevalent and incident vertebral fractures in osteoporosis research. Osteoporosis International 200314 S43-S55.

19 Duan Y, Parfitt MA \& Seeman E. Vertebral bone mass, size, and volumetric density in women with spinal fractures. Journal of Bone and Mineral Research 199914 1796-1802.

20 Carnevale V, Manfredi G, Romagnoli E, De Geronimo S, Paglia F, Pepe J, Scillitani A, D’Erasmo E \& Minisola S. Vitamin D status in female patients with primary hyperparathyroidism: does it play a role in skeletal damage? Clinical Endocrinology 200460 81-86.

21 Bilezikian JP \& Silverberg SJ. Asymptomatic primary hyperparathyroidism. New England Journal of Medicine $2004 \mathbf{3 5 0}$ 1746-1751.

22 Minisola S, Rosso R, Romagnoli E, Pepe J, De Geronimo S, Dionisi S, Paglia F, Raejentroph N, Aliberti G \& Mazzuoli GF. Uneven deficits in vertebral bone density in postmenopausal patients with primary hyperparathyroidism as evaluated by posterior-anterior and lateral dual-energy absorptiometry. Osteoporosis International 200213 618-623.

23 Bilezikian JP. Bone strength in primary hyperparathyroidism. Osteoporosis International 200314 S113-S117.

24 Parfitt MA. Parathyroid hormone and periosteal bone expansion. Journal of Bone and Mineral Research 200217 1741-1743.

25 Chen Q, Kaji H, Iu M-F, Nomura R, Sowa H, Yamauchi M, Tsukamoto T, Sugimoto T \& Chihara K. Effects of an excess and a deficiency of endogenous parathyroid hormone on volumetric bone mineral density and bone geometry determined by peripheral quantitative computed tomography in female subjects. Journal of Clinical Endocrinology and Metabolism $2003 \mathbf{8 8}$ 4655-4658.

26 Kaji H, Yamauchi M, Chihara K \& Sugimoto T. The threshold of bone mineral density for vertebral fractures in female patients with primary hyperparathyroidism. European Journal of Endocrinology 2005153 373-378.

27 Zanchetta JR, Bogado CE, Ferretti JL, Wang O, Wilson MG, Sato M, Gaich GA, Dalsky GP \& Myers SL. Effects of teriparatide (recombinant human parathyroid hormone (1-34)) on cortical bone in postmenopausal women with osteoporosis. Journal of Bone and Mineral Research 200318 539-543.

28 Nordenström E, Westerdahl J, Lindergard B, Lindblom P \& Bergenfelz A. Multifactorial risk profile for bone fractures in primary hyperparathyroidism. World Journal of Surgery 200226 1463-1467.

29 Bischoff-Ferrari HA. Extra-skeletal effects of vitamin D. Current Opinion in Endocrinology and Diabetes 20052 464-470.

Received 17 March 2006

Accepted 7 June 2006 